\title{
GHz Laser-free Time-resolved Transmission Electron Microscopy: a Stroboscopic High-duty-cycle Method
}

\author{
Jiaqi Qiu, Gwanghui Ha, ${ }^{*}$ Chunguang Jing, Sergey V. Baryshev ${ }^{\dagger}$ \\ Euclid TechLabs, 365 Remington Blvd., Bolingbrook, IL 60440, USA
}

Bryan W. Reed

Integrated Dynamic Electron Solutions, 5653 Stoneridge Dr., Suite 117, Pleasanton, CA 94588, USA

June W. Lau

Materials Science and Engineering Division, National Institute of Standards and Technology,

Gaithersburg, MD 20899, USA

Yimei Zhu

Department of Condensed Matter Physics and Materials Science, Brookhaven National Laboratory, Upton, NY 11973, USA

\begin{abstract}
.
A device and a method for producing ultrashort electron pulses with $\mathrm{GHz}$ repetition rates via pulsing an input direct current (dc) electron beam are provided. The device and the method are based on an electromagnetic-mechanical pulser (EMMP) that consists of a series of transverse deflecting cavities and magnetic quadrupoles. The EMMP modulates and chops the incoming dc electron beam and converts it into pico- and sub-pico-second electron pulse sequences (pulse trains) at $>1 \mathrm{GHz}$ repetition rates, as well as controllably manipulates the resulting pulses. Ultimately, it leads to negligible electron pulse phase-space degradation compared to the incoming dc beam parameters. The temporal pulse length and repetition rate for the EMMP can be continuously tunable over wide ranges.
\end{abstract}

Applying the EMMP to a transmission electron microscope (TEM) with any dc electron source (e.g. thermionic, Schottky, or field-emission source), a GHz stroboscopic high-duty-cycle TEM can be realized. Unlike in many recent developments in time-resolved TEM that rely on a sample pumping laser paired with a laser launching electrons from a photocathode to probe the sample, there is no laser in the presented experimental set-up. This is expected to be a significant relief for electron microscopists who are not familiar with laser systems. The EMMP and the sample are externally driven by a radiofrequency (RF) source synchronized through a delay line. With no laser pumping the sample, the problem of the pump laser induced residual heating/damaging the sample is eliminated. As many RF-driven processes can be cycled indefinitely, sampling rates of 1-50 GHz become accessible. Such a GHz stroboscopic TEM would open up a new paradigm for in situ and in operando experiments to study samples externally driven electromagnetically. Complementary to the lower $(\mathrm{MHz})$ repetition rates experiments enabled by laser photocathode TEM, new experiments in

\footnotetext{
${ }^{*}$ Also with POSTECH, Korea

†s.baryshev@euclidtechlabs.com / sergey.v.baryshev@gmail.com
} 
the high rep-rate multi-GHz regime will be enabled by the proposed RF design. Because TEM is also a platform for various analytical methods, there are infinite application opportunities in energy and electronics to resolve charge (electronic and ionic) transport, and magnetic, plasmonic and excitonic dynamics in advanced functional materials. In addition, because the beam duty-cycle can be as high as $\sim 10^{-1}$ (or $10 \%$ ), detection can be accomplished by commercially available detectors.

In this article, we report an optimal design of the EMMP. The optimal design was found using an analytical generalized matrix approach in the thin lens approximation along with detailed beam dynamics taking actual realistic dc beam parameters in a TEM operating at $200 \mathrm{keV}$.

\section{Introduction}

The past 10 years has seen enormous advancement in time-resolved transmission electron microscopes (TEMs), driven largely by advances in pulsed laser systems. Timeresolved ultrafast TEM (UTEM) [1] and dynamic TEM (DTEM) [2] are steadily establishing new measurement capabilities to observe, understand and control solid or soft materials at the most basic level under equilibrium, far-from-equilibrium, extreme, or in situ and in operando conditions. In a broader context, UTEM and DTEM are electron methods to probe structural dynamics. Even though electrons interact with matter $10^{5}$ times more strongly than photons (electrons are $\sim 10^{5}$ stronger in scattering power than photons), ultrafast science instrumentation is currently dominated by synchrotron x-ray and x-ray free electron laser facilities, as well as by the use of rare and highly customized high harmonic generation laser microscopes. Since electron- and photon-based tools are fundamentally different, a comparison can be drawn using a combined resolution metric called space-time resolution (STR, spatial resolution times temporal resolution) - in both classes STR can be $\leq 10^{-20} \mathrm{~m} \cdot \mathrm{s}$. Nevertheless, there are two other parameters that are important: the sampling rate (repetition rate) and beam duty cycle of the electron or photon pulse sequences. In the modern technological era, many breakthroughs rely on understanding how advanced nanoscopic materials or devices operate at rates approaching or exceeding $1 \mathrm{GHz}$. For example, spintronics exploit ferromagnetic resonance in magnetic materials which occurs in the $\mathrm{GHz}$ regime [3]. Understanding how magnetic eigenmodes and magnons interact with real material, defects, and interfaces is a major impendent to the advancement of spintronics. Another example is MEMS/NEMS, which exploit mechanical oscillation of micro-/nanostructures in the $\mathrm{MHz}$ and $\mathrm{GHz}$ [4]. In operando measurements of mechanical properties of these structures will lead to breakthroughs for smaller and faster devices. These examples illustrate the need for adequate instrumentation. At the moment, none of the aforementioned techniques/tools is able to provide $\mathrm{GHz}$-scale sampling rates.

Factors limiting sampling rates at light sources are complex, and even if they are solved, limited beam time allocations will still limit ubiquity of x-ray $\mathrm{GHz}$ methods. In contrast, UTEM and ultrafast electron diffraction [5] approaches can be developed at the scale of single principal investigators, and in this case the barrier to $\mathrm{GHz}$ sampling arises from the inherent limitations of driving processes with a pump laser. UTEM is a stroboscopic pump- 
probe method in which data are repeatedly collected over extended periods of time and thermal load from the pump laser must be handled so a process under study stays reversible [6]. Therefore, even though lasers with higher repetition rates are available, UTEM systems typically operate at much less than $0.1 \mathrm{GHz}$, and sometimes even at $\sim 0.1 \mathrm{MHz}$, depending on the experiment. In contrast to most laser-driven processes, many processes driven electrically/magnetically or both can be cycled indefinitely at $\mathrm{GHz}$ frequencies, one example being switching in a semiconductor device. This class of problems, characterized by electrical stimulus and lack of an extended cool-down time, is largely distinct from the class of problems typically studied in laser-based UTEM systems. Thus a purely electrical approach to pump/probe electron microscopy can complement existing laser-based approaches by reaching much higher repetition rates in the study of processes that can be so driven. Higher repetition rates have an immediate advantage in terms of the amount of time required to accumulate a measured signal because of the potential for much higher duty cycles and thus much higher time-averaged probe current.

As an alternative to the laser-photocathode combination method of producing electron pulses, blanking of a direct current (dc) electron beam can produce periodic electron pulse sequences with a flexible temporal structure that can be perfectly synchronized with the clock signal driving a high-frequency nanoscale device (be it a transistor, a plasmonic laser diode, a nano-electromechanical system, a spin-transfer torque memory, or any other such device). The basic principle of such stroboscopic TEM is presented in Fig.1. A small part of the RF signal to the dc beam pulsing device will be diverted to the sample through a phaselocked delay line. Similar to UTEM, issues like heating can be anticipated, but even so, essential difference here is that near- in operando examination of devices or device structures is feasible, since their realistic operation is itself electromagnetically driven and long thermal cool-down can be avoided. More specifically, such a microscope would reveal the inner workings of such devices in unprecedented ways, by bringing all of the highresolution imaging, analytical, nanoscale diffraction (including strain measurements), and other capabilities (such as holographic imaging of electric fields or spectroscopic imaging of plasmonic fields combined with tomography, making it truly 4D) of a modern TEM down to the time scale of the device's normal operation. In the method being proposed, the pulsed laser system is eliminated, which significantly reduces system complexity and enhances the ease of use. These are important factors to consider when studying already highlycomplicated systems for high-technology applications. 


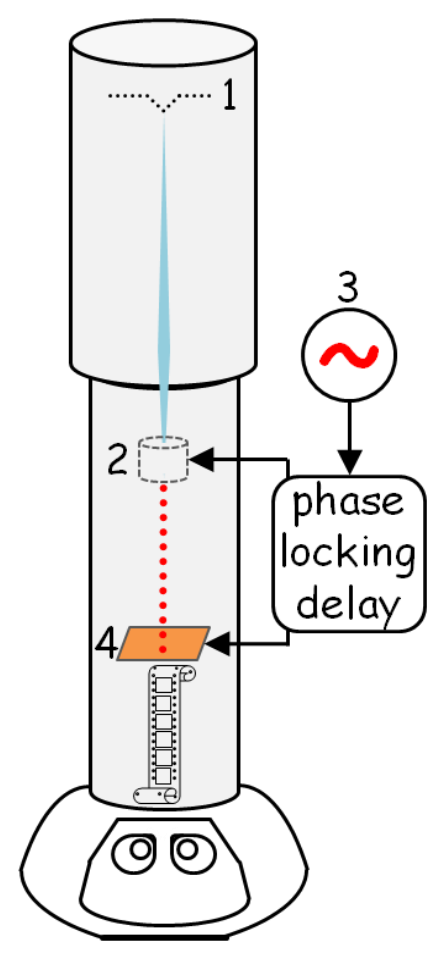

Fig.1. Basic principle of the stroboscopic laser-free high-duty cycle TEM: 1 is the electron dc source; 2 is a dc beam pulsing device; 3 is an RF source; 4 is the sample.

The first prototypes of time-resolved electron microscopes operating in a stroboscopic regime via blanking the dc beam were dates back to the 1960s. Those were scanning electron microscopes (SEM) used to image electron current propagation in novel (for that time) semiconductor devices such as MOSFETs [7] and Gunn-effect devices [8]. The achievable frame size (single pulse length) and frame (repetition) rate parameters were driven by progress in semiconductor technology - from MHz in 1968 [7] to $\mathrm{GHz}$ in 1978 [8]. Thus, by early 1980s stroboscopic SEMs and TEMs looking into processes with temporal resolution as low as $10 \mathrm{ps}$ were widely prototyped. While ns-pulses at $\mathrm{MHz}$ repetition rates were easy to generate using a standard deflecting plate system [7], ps-pulses at $\mathrm{GHz}$ repetition rates required developments of new strategies, which included a specialty meander travelling-wave line [9], specialty fast capacitors [10], and an RF pillbox cavity with a deflecting mode operating at $1 \mathrm{GHz}$ applied for practical use in electron microscopy for the first time [8]. The aforementioned examples made use of SEMs with lateral resolution just below 1 micron and very early-generation TEMs with spatial resolution much worse than in current instruments. While imaging with spatial resolution between $1 \AA$ and 1 $\mathrm{nm}$ is routine in a modern TEM, recent interest in time-resolved and pump-probe experiments sets much more challenging requirements for electron microscopy: additional temporal resolution between $10 \mathrm{~ns}$ and $<100 \mathrm{fs}$ is now expected on top of the same spatial resolution range. 
None of the existing time-resolved microscopes have simultaneously achieved all three of the following parameters: (1) Continuously tunable pulse duration between $\sim 100 \mathrm{fs}$ and $\sim 100 \mathrm{ps}$; (2) Continuously tunable rates of repetition from $\sim 0.1 \mathrm{GHz}$ to $\sim 50 \mathrm{GHz}$; (3) Phasespace fidelity of electron pulses so that processes at nanometer length scales and below can be resolved. By continuously tunable we mean that these parameters can be adjusted smoothly throughout their defined ranges, with no gaps. In this paper, we describe a design of a compact, versatile, drop-in dc beam pulsing device for medium electron energies $(\sim 100$ $\mathrm{keV}$ ) to enable laser-free $\mathrm{GHz}$ stroboscopic TEM. The final design was chosen from a number of candidates based on the results of analytical geometrical optics approximation and detailed beam dynamics calculations. The solution is such that when the dc beam pulsing device is ON, only electrons matching the strobe frequency are allowed access to the sample and the TEM operates in the GHz stroboscopic mode. When the dc beam pulsing device is OFF, the TEM operates in its conventional dc beam mode.

\section{Approaches and methods: basic design of the dc beam pulsing device}

To pulse the dc beam, mechanical or electromagnetic approaches can be used. Both mechanical shaping and/or electromagnetic manipulations of electron pulsed beams are common in the field of particle accelerators. They are used to tailor electron beam properties and for electron beam diagnostics, including emittance exchange experiments [11], phasespace imaging [12], energy-chirp compensation [13] and accelerator-based radiation sources [14, 15]. Particularly, electromagnetic RF cavities are gaining more applications in timeresolved electron microscopes/beam-lines being utilized as electron RF guns [16] and electron pulse compressors $[6,17,18]$. Following previous reports on designing a combined electromagnetic-mechanical pulser (dubbed as EMMP) operated at GHz rates [8, 19], the core component consists of a transverse deflecting cavity (TDC) and a chopping collimating aperture (CCA). A concept of this device is shown in Fig.2. A vacuum TDC, externally driven by an RF source, operated in TM110 mode at $f_{0}=10 \mathrm{GHz}$ (corresponding to a diameter of $39 \mathrm{~mm}$ ) and a CCA are used to form ultrahigh repetition rate pulse sequences (the repetition rate here is $20 \mathrm{GHz}$ because pulses are produced by cutting both sides, leading and trailing, of the sinusoid). At the fixed fundamental TDC frequency of $10 \mathrm{GHz}$, the pulse length can be continuously changed between $100 \mathrm{fs}$ and $10 \mathrm{ps}$ by varying the CCA diameter and/or RF power in the cavity. The exact range of duty cycle depends on the ratio of the diameters of the TDC (determining $f_{0}$ ) and the CCA, and the power fed by the RF source into the TDC. For the TM110 mode in a pillbox, a general relation between all the parameters involved is described as

$$
P \propto B^{2}=\frac{r \times m_{e}}{d \times e \times \Delta t},
$$

where $P$ and $B$ are power and magnetic component of the electromagnetic field in the TDC, respectively; $m_{e}$ and $e$ are the electron mass and charge, respectively; $r$ is the radius of the 
CCA; $d$ is the free-drifting distance between the TDC and the CCA; and $\Delta t$ is the electron pulse length. This leads to duty cycles of up to $2 \times 10^{-1}$ (or $20 \%$ ). One important note: the RF pillbox cavity technology is downwards compatible to sampling rate (or strobe rate) in the $\mathrm{MHz}$ by replacing vacuum in the TDC with a high permittivity dielectric. The general relation linking the TDC diameter $(D)$, the fundamental TDC frequency $\left(f_{0}\right)$ and the permittivity $(\varepsilon)$ is $D \sim \frac{1}{f_{0} \times \sqrt{\varepsilon}}$. With a high permittivity ferroelectric, the TDC can be continuously tunable too in a specific frequency range. We present the case where the TDC is operated at fundamental frequency of $10 \mathrm{GHz}$ (equivalent to $20 \mathrm{GHz}$ repetition rate) in this study because such a sampling rate can satisfy a multitude of ultrafast experiments (e.g. nanoscale magnetic dynamics). At the same time, this example illustrates the challenges in preserving remarkable quality of the unmodified dc beam like the original energy spread. Lower sampling rates at longer pulse lengths will always have more benign effects on the pulsed electron beam.

The incoming longitudinal electron dc beam traveling along the $z$-axis (Fig.2), blue straight segment originating before $z=0 \mathrm{~cm}$ ) picks up a transverse sinusoidal momentum by the electromagnetic (EM) field in the TDC. Since the EM field oscillates with the radial frequency $f_{0}$, the modulation force depends on the time at which electrons arrive in the TDC. The amplitude of the sinusoid grows as the modulated beam propagates. The CCA is placed on axis to chop the beam and converts it into a pulse sequence. As mentioned in earlier publications on the stroboscopic pulsers/choppers, different scenarios of spatial deterioration of the electron beam were observed $[8,19]$. Indeed, using the concept in Fig.2 the beam would expand in the transverse (beam diameter growth) and longitudinal (temporal coherence deterioration) directions after drifting a certain distance. While this was not an issue for imaging on the micron scale, much more rigorous schemes must be considered in order to ensure beam spatial and energy coherence necessary for TEM spectroscopy, diffraction and imaging. Thus, additional optical elements are required downstream of the CCA to mitigate the effects produced by the first TDC. These additional elements are represented as blank squares in Fig.2, which will be determined in sections 3 and 4 . 


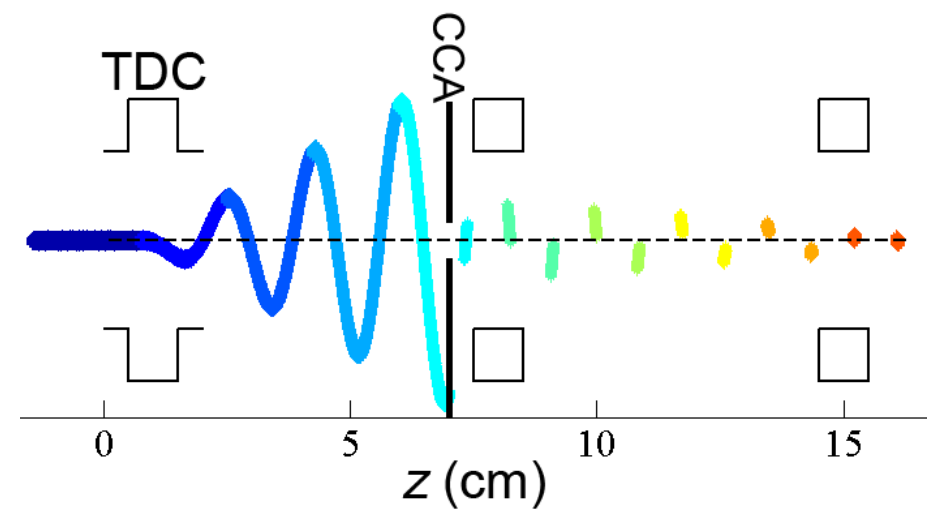

Fig.2. General concept of the compact electromagnetic-mechanical pulser (EMMP) for pulsing dc electron beams and manipulating electron pulses in the phase space. (The $z$-scale is approximate, and color coding is arbitrary.) Additional elements (shown here as generic squares representing either two TDCs or a magnetic quadrupole and a second TDC) after the CCA compensate for the introduced transverse momentum, resulting in a beam with very nearly the same transverse phase space profile as in normal operation by the time it exits the final TDC at the right-hand side of the diagram.

\section{Generalized matrix calculations}

Since the final goal is integration with a TEM, the EMMP should be as compact as possible. This not only places an upper limit on the number of optical elements, but also makes it desirable to minimize the number of necessary adjustments to tune the EMMP. To come up with the best miniature design for removing the post-TDC distortions of the resulting electron pulses, we use a generalized matrix approach. In this approach, optical elements are described by matrices. Matrix components depend on the actual geometry of the EMMP, and the strength of various effects on electron dynamics in the phase space can be predicted and evaluated. The matrix methodology relies on three basic assumptions: (1) electron optics elements are approximated as thin lenses; (2) it considers a single particle/electron; (3) only linear matrix transformations are considered. All three assumptions are intertwined. When combined, they establish the basis for the geometrical optics framework in which the problem is solved. This idealized framework provides a good first-order model for rapid progress in the design, to be followed up with full ray-trace calculations including space charge effects to determine the effects of aberrations and undesired couplings on the electron phase space.

An initial and a final state of an electron at input and at the output of the EMMP are linked in the momentum-coordinate phase space via a beam transport matrix as follows 


$$
\left(\begin{array}{l}
x_{f} \\
x_{f}^{\prime} \\
y_{f} \\
y_{f}^{\prime} \\
z_{f} \\
\frac{\Delta p_{f}}{p_{0}}
\end{array}\right)=\left(\begin{array}{llllll}
R_{11} & R_{12} & R_{13} & R_{14} & R_{15} & R_{16} \\
R_{21} & R_{22} & R_{23} & R_{24} & R_{25} & R_{26} \\
R_{31} & R_{32} & R_{33} & R_{34} & R_{35} & R_{36} \\
R_{41} & R_{42} & R_{43} & R_{44} & R_{45} & R_{46} \\
R_{51} & R_{52} & R_{53} & R_{54} & R_{55} & R_{56} \\
R_{61} & R_{62} & R_{63} & R_{64} & R_{65} & R_{66}
\end{array}\right)\left(\begin{array}{l}
x_{i} \\
x_{i}^{\prime} \\
y_{i} \\
y_{i}^{\prime} \\
z_{i} \\
\frac{\Delta p_{i}}{p_{0}}
\end{array}\right),
$$

where $x$ is the relative horizontal beam position, $x^{\prime}$ is the horizontal divergence, $y$ is the relative vertical beam position, $y^{\prime}$ is the vertical divergence, $z$ is the relative longitudinal position (principal optical axis of the TEM) or time, and $\frac{\Delta p}{p_{0}}$ is the relative longitudinal momentum.

In Eq.2, the matrix $R(6 \times 6)$ is called the transport matrix. It is a result of multiplication of all matrices describing every single component of an EMMP design; this includes the drifting matrix describing empty gaps/pipes between hardware components. The perfect case is when the matrix $R$ has only diagonal elements: it means an electron beam transformation took place, yet cross-correlations, described by off-diagonal elements resulting in pulse size change in transverse and longitudinal directions and energy spread, are absent. A number of combinations and designs were analyzed, and the main conclusion was that at least 3 active elements must be present to minimize off-diagonal elements. While the first element is always a TDC, second and third elements are shown as blank squares in Fig.2, and should be determined from the matrix analysis. It was found that best results can be anticipated when combinations of TDCs and/or magnetic quadrupoles (MQs) are used, provided such combinations are also most compact and simple from the operational point of view. Two most attractive designs are illustrated in Fig.3. 


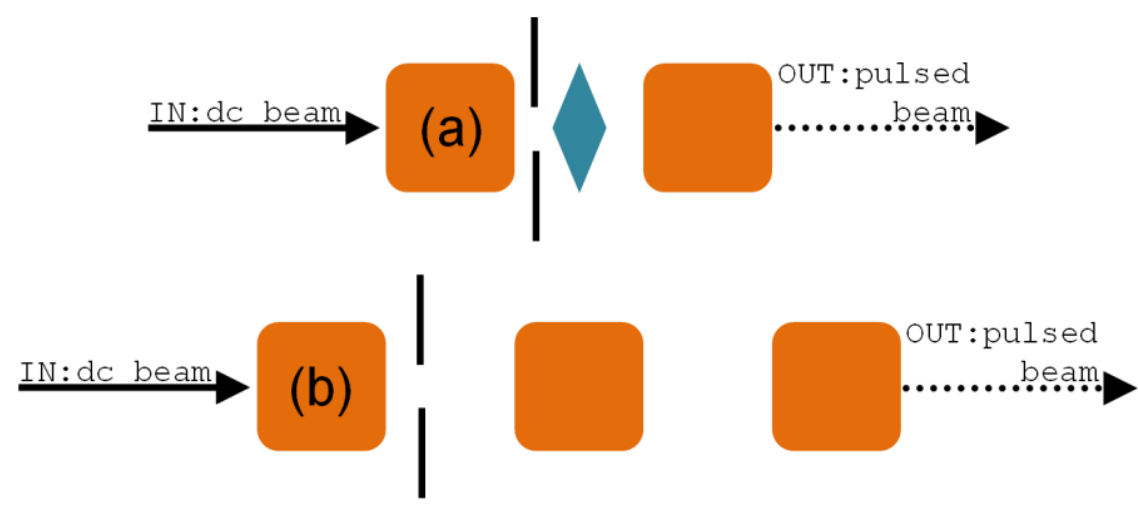

Fig.3. Two main EMMP designs for further considerations based on the matrix analysis (not to scale).

The orange square is the TDC, and the cyan diamond is the MQ. Design (a) is abbreviated as TDC+MQ+TDC and design (b) is abbreviated as 3TDC.

Thus, the resulting matrix $R(6 \times 6)$ is the product of 5 -fold multiplication of 3 key matrices related to the EMMP designs in Fig.3. Free drift beam pipe of length $d$ (empty space between either optical component in the EMMP) [measured in meters] is described by the following matrix

$$
\left(\begin{array}{llllll}
1 & d & 0 & 0 & 0 & 0 \\
0 & 1 & 0 & 0 & 0 & 0 \\
0 & 0 & 1 & d & 0 & 0 \\
0 & 0 & 0 & 1 & 0 & 0 \\
0 & 0 & 0 & 0 & 1 & \frac{d}{\gamma^{2}} \\
0 & 0 & 0 & 0 & 0 & 1
\end{array}\right),
$$

where $\gamma$ is the Lorentz factor. Its value depends on the electron energy. The magnetic quadrupole with a focal length $f$ [measured in meters] is described as

$$
\left(\begin{array}{cccccc}
1 & 0 & 0 & 0 & 0 & 0 \\
\frac{1}{f} & 1 & 0 & 0 & 0 & 0 \\
0 & 0 & 1 & 0 & 0 & 0 \\
0 & 0 & -\frac{1}{f} & 1 & 0 & 0 \\
0 & 0 & 0 & 0 & 1 & 0 \\
0 & 0 & 0 & 0 & 0 & 1
\end{array}\right) .
$$

The TDC has a matrix 


$$
\left(\begin{array}{llllll}
1 & 0 & 0 & 0 & 0 & 0 \\
0 & 1 & 0 & 0 & k & 0 \\
0 & 0 & 1 & 0 & 0 & 0 \\
0 & 0 & 0 & 1 & 0 & 0 \\
0 & 0 & 0 & 0 & 1 & 0 \\
k & 0 & 0 & 0 & 0 & 1
\end{array}\right),
$$

where $k$ is the transverse momentum acquired by an electron in the TDC, and it is measured in reciprocal meters. In what follows, $k$ will be referred to as kick. Resulting transport matrices for the designs, sketched in Fig.3, were optimized in order to zero as many offdiagonal elements as possible. For this calculation, the following dc beam input parameters were considered: (1) beam energy $\left(\mathrm{E}_{0}\right) 200 \mathrm{keV}$; (2) energy spread $(\Delta \mathrm{E}) 0.5 \mathrm{eV}$; (3) emittance $1.5 \mathrm{~nm} \times \mathrm{rad}$ which was a product of a beam diameter of $10 \mu \mathrm{m}$ and a divergence angle of $0.15 \mathrm{mrad}$; these values are also typical for various accelerator applications. The TDC+MQ+TDC design (Fig.3a) has a transport matrix

$$
\left(\begin{array}{cccccc}
-\frac{d_{2}}{d_{1}} & 0 & 0 & 0 & 0 & 0 \\
-\frac{\left(\gamma^{2}-d_{1}^{2} k_{1}^{2}\right)\left(d_{1}+d_{2}\right)}{d_{1} d_{2} \gamma^{2}} & -\frac{d_{1}}{d_{2}} & 0 & 0 & 0 & \frac{d_{1} k_{1}\left(d_{1}+d_{2}\right)}{d_{2} \gamma^{2}} \\
0 & 0 & 2+\frac{d_{2}}{d_{1}} & 2\left(d_{1}+d_{2}\right) & 0 & 0 \\
0 & 0 & \frac{d_{1}+d_{2}}{d_{1} d_{2}} & 2+\frac{d_{1}}{d_{2}} & 0 & 0 \\
\frac{k_{1}\left(d_{1}+d_{2}\right)}{\gamma^{2}} & 0 & 0 & 0 & 1 & \frac{d_{1}+d_{2}}{\gamma^{2}} \\
0 & 0 & 0 & 0 & 0 & 1
\end{array}\right)
$$

where $d_{1}$ and $d_{2}$ are the drift distances between the first TDC and the MQ, and between the MQ and the second TDC respectively; $k_{1}$ is the kick strength of the first deflecting cavity. The focal length of the MQ is $f=-\frac{d_{1} d_{2}}{d_{1}+d_{2}}$, and the kick strength of the second deflecting cavity is $k_{2}=\frac{d_{1}}{d_{2}} k_{1}$. For the 3TDC (Fig.3b) design, the $R$-matrix is

$$
\left(\begin{array}{cccccc}
1-\frac{d_{1}\left(d_{1}+d_{2}\right) k_{1}^{2}}{\gamma^{2}} & d_{1}+d_{2} & 0 & 0 & 0 & -\frac{d_{1}\left(d_{1}+d_{2}\right) k_{1}}{\gamma^{2}} \\
-\frac{d_{1}\left(d_{1}+d_{2}\right) k_{1}^{2}}{d_{2} \gamma^{2}} & 1+\frac{d_{1}^{2}\left(d_{1}+d_{2}\right) k_{1}^{2}}{d_{2}\left(d_{1}\left(d_{1}+d_{2}\right) k_{1}^{2}-\gamma^{2}\right)} & 0 & 0 & 0 & -\frac{d_{1}^{2}\left(d_{1}+d_{2}\right)^{2} k_{1}^{3}}{d_{2}\left(d_{1}^{2} k_{1}^{2}+d_{1} d_{2} k_{1}^{2}-\gamma^{2}\right)} \\
0 & 0 & 1 & d_{1}+d_{2} & 0 & 0 \\
0 & 0 & 0 & 1 & 0 & 0 \\
0 & -\frac{d_{1}\left(d_{1}+d_{2}\right) k_{1}}{\gamma^{2}} & 0 & 0 & 1-\frac{d_{1}\left(d_{1}+d_{2}\right) k_{1}^{2}}{\gamma^{2}} & \frac{d_{1}+d_{2}}{\gamma^{2}} \\
d_{2}\left(d_{1}^{2} k_{1}^{2}+d_{1} d_{2} k_{1}^{2}-\gamma^{2}\right) & 0 & 0 & -\frac{d_{1}\left(d_{1}+d_{2}\right) k_{1}^{2}}{d_{2}} & 1+\frac{d_{1}^{2}\left(d_{1}+d_{2}\right) k_{1}^{2}}{d_{2}\left(d_{1}\left(d_{1}+d_{2}\right) k_{1}^{2}-\gamma^{2}\right)}
\end{array}\right),
$$


where $k_{2}=-\frac{d_{1}+d_{2}}{d_{2}} k_{1}$ and $k_{3}=\gamma^{2} \frac{d_{1}}{d_{2}\left(\gamma^{2}-d_{1}\left(d_{1}+d_{2}\right) k_{1}^{2}\right)} k_{1}$ are found as optimal for the overall system design, i.e. maximum off-diagonal elements are zeros.

While the matrix methodology is widely used in high energy physics (electron beam energy range $\sim 10 \mathrm{MeV}$ ), it is challenging to apply it to the medium energy electron beams $(\sim 100 \mathrm{keV})$ found in TEMs due primarily to the Lorentz factor. Many off-diagonal elements, in the transport matrices (Eq. 6 and 7) are proportional to $\frac{1}{\gamma^{2}}$. So for a $10 \mathrm{MeV}$ beam where $\gamma \approx 20$, the off-diagonal elements become negligibly small, this is certainly not the case for a $100 \mathrm{keV}$ beam where $\gamma \approx 1$. From the matrices, it is seen that in transverse directions both designs may lead to satisfactory results such that sufficient spatial coherence in the beam is conserved upon EMMP installation. The main problem here is the matrix term $R_{65}$ which is responsible for energy spread growth. In this idealized geometrical optics framework, $R_{65}$ is zero for the TDC+MQ+TDC design, but is finite for the 3TDC design. That is, in the 3TDC case, the additional energy spread at $\mathrm{E}_{0}=200 \mathrm{keV}$ is higher than $1 \mathrm{eV}$ on top of the default/intrinsic energy spread of $0.5 \mathrm{eV}$.

\section{Beam dynamics simulations}

Matrix calculations provided a good starting point for the actual EMMP design. In the 3TDC case, it is seen that the energy spread is proportional to the kick value to the second power, $k_{1}^{2}$. Since the resulting electron pulse length is controlled by both $k_{1}$ and the CCA radius, to achieve a short pulse we must either have a large kick or a small aperture. Thus, to reduce energy spread for a given pulse size, we should choose a CCA radius as small as reasonably possible, thus allowing us to reduce the kick. At the same time, if the beam radius is larger than the CCA radius the EMMP will not be able deliver effective chopping at the CCA no matter how strong the kick is. Thus, the radius of the incoming dc beam produces a limitation for the EMMP in terms of the relation between the CCA radius and the kick strength, and consequently a limitation for minimal extrinsic/additional energy spread achievable upon pulsing. Beam dynamics calculations were carried out to compare transverse and longitudinal phase spaces between the input dc beam and the output pulsed beam. Using the geometry and beam characteristics described by Reed et al. [20] and keeping in mind the previously described limitations (dc beam radius versus CCA radius), we conducted our simulation in the soft focusing regime meaning that an input dc beam radius was $3 \mu \mathrm{m}$ and an angle of convergence was $0.1 \mathrm{mrad}$, resulting in beam emittance 0.3 $\mathrm{nm} \times \mathrm{rad}$. Changing the beam emittance between the default TEM mode and the soft focusing regime can be achieved by inserting a custom condenser lens (C0) similar to one described in Ref.[20], thus allowing a large fraction of the current emitted from the gun to be collected, collimated, and aligned into the pulser with a small convergence angle. Total dc current at $\mathrm{C} 0$ of $\sim 100 \mathrm{nA}$ is feasible in this alignment. The soft focusing is illustrated in Fig.4. Dotted 
red lines represent a way the dc beam should propagate and converge through the EMMP when it is OFF. Using C0 to create a soft crossover at the CCA plane allows optimal modulation and chopping upon turning the EMMP ON. Normal TEM operation with a lowemittance dc beam is obtained by turning off both $\mathrm{C} 0$ and the pulser components.

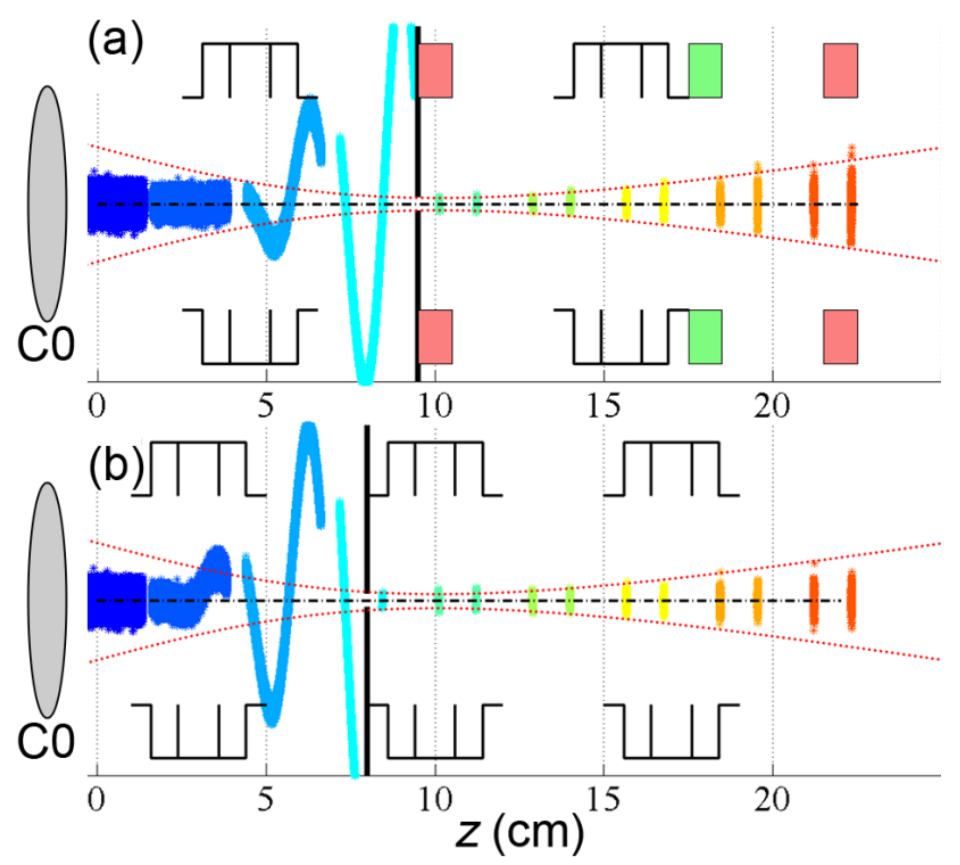

Fig.4. Two key designs identified by the matrix calculations and tested through beam dynamics. (a) TDC+MQ+TDC design; (b) 3TDC design. Red Dotted lines represent beam waist in the middle of the second active EMMP element, i.e. MQ (a) and TDC (b) - not to scale. The central black dashdotted line is the symmetry (optic) axis.

In terms of the device placement, a combined section containing C0 and EMMP should be inserted after the ultrahigh vacuum gun chamber but before the condenser lens section. This means operation and maintenance under the moderate column vacuum conditions which (1) completely circumvents the need to disturb the electron source and (2) preserves the factory packaging and design of the condenser system. After reviewing microscope raydiagrams, we found this was the best location for preserving all the available imaging and diffraction modalities. First, we emphasize that there is no change in dc beam propagation before the condenser lens system. Since beam phase-space manipulations to achieve different imaging modes in a TEM column take place at or below the condensers, by inserting the device between the gun and the condenser lenses, the problem is reduced to preserving the transverse and longitudinal phase spaces as the dc beam converts into a pulse train as it passes through the EMMP. Based on the soft focusing parameters, an input file representing the incoming dc beam was built using the code PARMELA [21, 22]. Input dc parameters and geometry used in the calculations are summarized in Table 1 and Fig.4, respectively. Particle tracking through the EMMP in the longitudinal and transverse phase 
spaces was performed by a custom-developed MATLAB [22] code. Resulting parameters are compiled in Table 1 . While both designs deliver nearly perfect conservation of the transverse phase space $\left(\varepsilon_{x}, \varepsilon_{y}\right)$, energy spread becomes a major parameter for design considerations. In TDC+MQ+TDC design, an energy spread as low as $0.25 \mathrm{eV}$ was achieved. Given a typical intrinsic energy spread in a TEM of $0.5 \mathrm{eV}$, the total value is $0.56 \mathrm{eV}$ for a $10 \mathrm{ps}$ pulse. As it was first suggested by matrix/thin lens approximations in Section 3, the TDC+MQ+TDC configuration (Figs.3a, 4a) is chosen for further hardware development based on this analysis. Such an energy resolution will allow pulsed electron energy-loss spectroscopy in TEM.

Table 1. Key design quantities (kick and CCA radius), and values characterizing the transverse and longitudinal phase spaces of the pulsed beam at the output of the EMMP with the two basic designs

(3TDC and TDC+MQ+TDC) considered.

\begin{tabular}{|c|c|c|c|c|c|c|}
\hline $\begin{array}{l}\text { EMMP } \\
\text { design }\end{array}$ & $\begin{array}{l}\text { pulse } \\
\text { length } \\
\text { (ps) }\end{array}$ & $\begin{array}{c}\text { kick } \\
(\mathrm{keV})\end{array}$ & $\begin{array}{l}\text { aperture } \\
\text { radius } \\
(\mu \mathrm{m})\end{array}$ & $\begin{array}{c}\varepsilon_{x} \\
(\mathrm{~nm} \times \mathrm{rad})\end{array}$ & $\begin{array}{c}\varepsilon_{y} \\
(\mathrm{~nm} \times \mathrm{rad})\end{array}$ & $\begin{array}{c}\text { energy } \\
\text { spread } \\
(\mathrm{eV})\end{array}$ \\
\hline INPUT & --- & --- & --- & 0.3 & 0.3 & --- \\
\hline \multirow{6}{*}{$\mathrm{TDC}+\mathrm{MQ}+\mathrm{TDC}$} & \multirow{4}{*}{1} & 1.25 & 5 & 0.24 & 0.39 & 0.81 \\
\hline & & 1.88 & 7.5 & 0.31 & 0.40 & 1.38 \\
\hline & & 2.50 & 10 & 0.38 & 0.43 & 2.23 \\
\hline & & 5.00 & 20 & 0.41 & 0.43 & 9.7 \\
\hline & \multirow{2}{*}{10} & 0.25 & 10 & 0.36 & 0.40 & 0.25 \\
\hline & & 0.50 & 20 & 0.38 & 0.39 & 1.01 \\
\hline \multirow{3}{*}{$3 \mathrm{TDC}$} & \multirow{2}{*}{1} & 2.5 & 10 & 0.34 & 0.42 & 28.4 \\
\hline & & 5 & 20 & 0.38 & 0.39 & 118 \\
\hline & 10 & 0.5 & 20 & 0.38 & 0.43 & 12.5 \\
\hline
\end{tabular}

In the TDC+MQ+TDC design, the MQ can be assembled with electro- or permanent magnets. Making use of a permanent magnet MQ would simplify the overall EMMP implementation. Taking it one step further, let us consider dc beam behavior when the EMMP is OFF. Fig.5 illustrates the root-mean-square (rms) transverse beam size, i.e. beam envelop in two transverse directions, with the EMMP ON (solid red and blue lines) and OFF (dotted red and blue lines). The state OFF means that only the TDCs are OFF, and the MQs are permanent magnets, i.e. $\mathrm{ON}$ all the time. It is seen that envelop and divergence after the aperture, especially at the outlet of the EMMP, are nearly identical. This result suggests that to return back to default TEM imaging mode with dc beam, in the TDC+MQ+TDC environment with the permanent magnets the RF source can be simply turned OFF such that TDCs become additional free drift space. If compact electromagnetic quadrupoles are in use, 
they can be either turned OFF such that the whole EMMP becomes a drift pipe and the dc beam is adjusted via $\mathrm{C} 0$, or the electromagnets are additionally used to steer and focus the dc beam, or they stay $\mathrm{ON}$ at the nominal values such that the situation in Fig. 5 takes place.

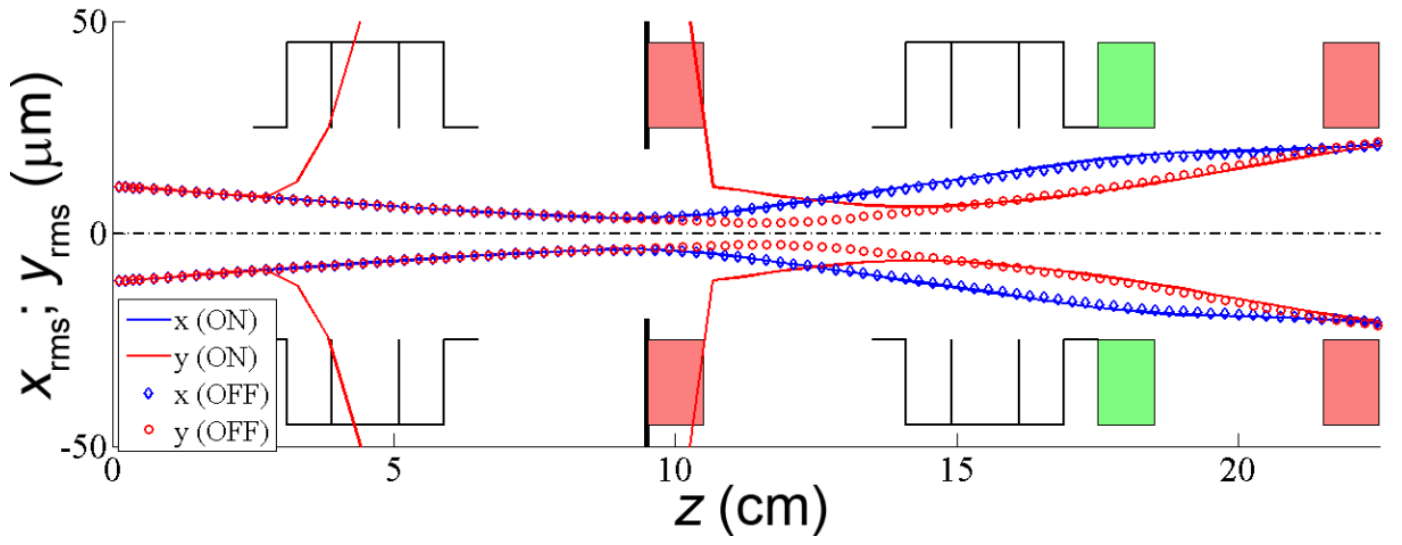

Fig.5. Beam envelop with the EMMP ON (solid lines) and OFF (dotted lines) - not to scale. Apparent discontinuity in the red solid curve is the result of RF modulation of the dc beam. The central black dash-dotted line is the symmetry (optic) axis.

\section{Conclusions}

To summarize, a novel time-resolved $\mathrm{GHz}$ stroboscopic concept for transmission electron microscopy is proposed. Such concept is laser-free and is aimed at resolving subnanosecond processes in advanced magnetic, electronic, ionic and photonic materials under actual operation conditions. A sample is given a $\mathrm{GHz}$ excitation with a radiofrequency source which is phase-locked through a delay line to an RF cavity pulser, which converts a standard dc beam in a TEM into pulse trains being driven by the same radiofrequency source. In the family of time-resolved electron probe methods, the laserfree/electromagnetically-driven $\mathrm{GHz}$ stroboscopic concept fulfills a different temporal landscape that is complementary to the existing commercial solutions. We used an analytical matrix algorithm and beam dynamics simulations to identify and rationalize a design-ofchoice for a compact pulser for a $200 \mathrm{keV}$ electron beam compatible with TEM columns. Table 2 summarizes key performance parameters anticipated in the proposed $\mathrm{GHz}$ stroboscopic TEM. 
Table 2. Summary of parameters considered in the paper.

\begin{tabular}{|c|c|c|}
\hline initial beam energy & \multicolumn{2}{|c|}{$200 \mathrm{keV}$} \\
\hline intrinsic energy spread & \multicolumn{2}{|c|}{$0.5 \mathrm{eV}$} \\
\hline dc beam current at gun exit & \multicolumn{2}{|c|}{ stroboscopic } \\
\hline operation mode & \multicolumn{2}{|c|}{ not required } \\
\hline laser & $1 \mathrm{ps}$ & $10 \mathrm{ps}$ \\
\hline pulse length & $20 \mathrm{GHz}$ & $20 \mathrm{GHz}$ \\
\hline repetition rate at specimen & $2 \times 10^{-2}(2 \%)$ & $2 \times 10^{-1}(20 \%)$ \\
\hline duty cycle & $\sim 1$ & $\leq 0.4 \mathrm{~nm} \times \mathrm{rad}$ \\
\hline number of electrons per cycle & $\leq 0.4 \mathrm{~nm} \times \mathrm{rad}$ & $0.25 \mathrm{eV}$ \\
\hline rms emittance & $0.81 \mathrm{eV}$ & $0.56 \mathrm{eV}$ \\
\hline extrinsic rms energy spread & $0.95 \mathrm{eV}$ & $2.80 \times 10^{-6}$ \\
\hline total energy spread & $4.75 \times 10^{-6}$ & $<10^{-20} \mathrm{~m} \cdot \mathrm{s}$ \\
\hline total relative energy spread & $<10^{-21} \mathrm{~m} \cdot \mathrm{s}$ & \\
\hline STR & & \multicolumn{2}{|c|}{} \\
\hline
\end{tabular}

\section{Acknowledgments}

Euclid TechLabs work was supported by DOE SBIR program grant No. DE-SC0013121. Y.Z. was supported by the U.S. Department of Energy, Office of Science, Office of Basic Energy Sciences, under Contract No. DE-SC0012704.

\section{References}

[1] D.J. Flannigan, A.H. Zewail, 4D Electron Microscopy: Principles and Applications, Accounts of Chemical Research, 45 (2012) 1828-1839.

[2] T. LaGrange, M.R. Armstrong, K. Boyden, C.G. Brown, G.H. Campbell, J.D. Colvin, W.J. DeHope, A.M. Frank, D.J. Gibson, F.V. Hartemann, J.S. Kim, W.E. King, B.J. Pyke, B.W. Reed, M.D. Shirk, R.M. Shuttlesworth, B.C. Stuart, B.R. Torralva, N.D. Browning, Single-shot dynamic transmission electron microscopy, Applied Physics Letters, 89 (2006) 044105.

[3] C. Kittel, On the Theory of Ferromagnetic Resonance Absorption, Physical Review, 73 (1948) 155-161.

[4] V. Cimalla, J. Pezoldt, O. Ambacher, Group III nitride and SiC based MEMS and NEMS: materials properties, technology and applications, Journal of Physics D: Applied Physics, 40 (2007) 6386-6434.

[5] P. Zhu, Y. Zhu, Y. Hidaka, L. Wu, J. Cao, H. Berger, J. Geck, R. Kraus, S. Pjerov, Y. Shen, R.I. Tobey, J.P. Hill, X.J. Wang, Femtosecond time-resolved MeV electron diffraction, New Journal of Physics, 17 (2015) 063004.

[6] A. Gliserin, S. Lahme, M. Walbran, F. Krausz, P. Baum, Ultrafast Single-Electron Diffraction, Ultrafast Phenomena XIX, 162 (2015) 295-298. 
[7] G.S. Plows, W.C. Nixon, Stroboscopic scanning electron microscopy, Journal of Physics E: Scientific Instruments, 1 (1968) 595-600.

[8] T. Hosokawa, H. Fujioka, K. Ura, Gigahertz stroboscopy with the scanning electron microscope, Review of Scientific Instruments, 49 (1978) 1293.

[9] A. Gopinath, M.S. Hill, Deflection beam-chopping in the SEM, Journal of Physics E: Scientific Instruments, 10 (1977) 229-236.

[10] H. Sadorf, H.A. Kratz, Plug-in fast electron beam chopping system, Review of Scientific Instruments, 56 (1985) 567.

[11] P. Emma, Z. Huang, K.J. Kim, P. Piot, Transverse-to-longitudinal emittance exchange to improve performance of high-gain free-electron lasers, Physical Review Special Topics Accelerators and Beams, 9 (2006) 100702.

[12] N.A. Yampolsky, Manipulations of the beam phase space with transverse deflecting cavities, 16th Advanced Accelerator Concepts Workshop, (2014).

[13] S. Antipov, S. Baturin, C. Jing, M. Fedurin, A. Kanareykin, C. Swinson, P. Schoessow, W. Gai, A. Zholents, Experimental Demonstration of Energy-Chirp Compensation by a Tunable Dielectric-Based Structure, Physical Review Letters, 112 (2014) 114801.

[14] Y.-C. Du, W.-H. Huang, C.-X. Tang, A new method to generate relativistic comb bunches with tunable subpicosecond spacing, Chinese Physics C, 36 (2012) 151-155.

[15] S. Antipov, M. Babzien, C. Jing, M. Fedurin, W. Gai, A. Kanareykin, K. Kusche, V. Yakimenko, A. Zholents, Subpicosecond Bunch Train Production for a Tunable mJ Level THz Source, Physical Review Letters, 111 (2013) 134802.

[16] R.K. Li, P. Musumeci, Single-Shot MeV Transmission Electron Microscopy with Picosecond Temporal Resolution, Physical Review Applied, 2 (2014) 024003.

[17] T. van Oudheusden, P.L.E.M. Pasmans, S.B. van der Geer, M.J. de Loos, M.J. van der Wiel, O.J. Luiten, Compression of Subrelativistic Space-Charge-Dominated Electron Bunches for Single-Shot Femtosecond Electron Diffraction, Physical Review Letters, 105 (2010) 264801.

[18] G.F. Mancini, B. Mansart, S. Pagano, B. van der Geer, M. de Loos, F. Carbone, Design and implementation of a flexible beamline for fs electron diffraction experiments, Nuclear Instruments and Methods in Physics Research Section A: Accelerators, Spectrometers, Detectors and Associated Equipment, 691 (2012) 113-122.

[19] A. Lassise, P.H.A. Mutsaers, O.J. Luiten, Compact, low power radio frequency cavity for femtosecond electron microscopy, Review of Scientific Instruments, 83 (2012) 043705.

[20] B.W. Reed, T. LaGrange, R.M. Shuttlesworth, D.J. Gibson, G.H. Campbell, N.D. Browning, Solving the accelerator-condenser coupling problem in a nanosecond dynamic transmission electron microscope, Review of Scientific Instruments, 81 (2010) 053706.

[21] L.M. Young, PARMELA, Los Alamos National Laboratory report LA-UR-96-1835, (2003).

[22] Certain commercial equipment, instruments, or materials are identified in this paper to specify the experimental procedure adequately. Such identification is not intended to imply recommendation or endorsement by the National Institute of Standards and Technology, nor is it intended to imply that the materials or equipment identified are necessarily the best available for the purpose. 